\title{
TET1 partially mediates HDAC inhibitor-induced suppression of breast cancer invasion
}

\author{
HOU-GEN LU ${ }^{1 *}$, WANG ZHAN ${ }^{2 *}$, LIN YAN ${ }^{1}$, RUI-YING QIN ${ }^{3}$, YI-PENG YAN ${ }^{3}$, ZHEN-JIANG YANG ${ }^{3}$, \\ GUI-CHAO LIU ${ }^{4}$, GUI-QIN LI ${ }^{3}$, HAI-FENG WANG ${ }^{5}$, XING-LIANG $\mathrm{LI}^{6}, \mathrm{ZHI} \mathrm{LI}^{6}$, $\mathrm{LU} \mathrm{GAO}^{7}$ and GUO-QING $\mathrm{CHEN}^{8}$ \\ ${ }^{1}$ Department of Orthopedics, Central Hospital of Jingzhou, Hubei 434100; ${ }^{2}$ Department of Medical Oncology, \\ Shanghai Changzheng Hospital, Shanghai 200070; ${ }^{3}$ The First Affiliated Hospital of Xin-Xiang Medical University, \\ Weihui, Henan 453100; ${ }^{4}$ Department of Orthopedics, Orthopaedics Hospital of Nanyang, Nanyang, Henan 473003; \\ ${ }^{5}$ Shanghai Hengrui Pharmaceutical Co., Ltd, Shanghai 20035; ${ }^{6}$ Shanghai Xuhai Biological Technology Co., Ltd, Shanghai 20032; \\ ${ }^{7}$ Department of Pharmaceutical Biotechnology, College of Life Sciences, Northeast Agricultural University, Harbin, \\ Heilongjiang 150030; ${ }^{8}$ Department of Surgery, Shanghai Tongren Hospital of Changning, Shanghai 200050, P.R. China
}

Received July 25, 2013; Accepted February 4, 2014

DOI: $10.3892 / \mathrm{mmr} .2014 .2517$

\begin{abstract}
Histone deacetylases (HDACs) are important in chromatin remodeling and epigenetic regulation of gene expression. Histone deacetylase inhibitors (HDACi) have highly effective anti-metastatic and anti-angiogenic activity in various types of cancer, while the molecular mechanisms involved in this process are not fully understood. In the present study, trichostatin A (TSA), a HDACi, was found to suppress MCF-7 breast carcinoma cell invasion and upregulate TET1 expression in a dose-dependent manner. TET1, a dioxygenase involved in cytosine demethylation, is downregulated during breast cancer progression. TET1 knockdown in MCF-7 cells facilitates cell invasion, inhibits the expression of tissue inhibitors of metalloproteinase 2/3 (TIMP2/3) and promotes matrix metalloproteinases (MMP) 2/9 transcriptional activity. Importantly, TET1 depletion impaired the inhibitory effect of TSA on breast cancer cell invasion. Together, these results illustrated a mechanism by which TET1 partially mediates HDACi elicited suppression of breast cancer invasion.
\end{abstract}

\section{Introduction}

Tumor invasion and metastasis are the main characteristics of various types of aggressive human cancer, including breast cancer (1). Cell invasion is one of the initiation steps for the metastatic cascade, during which cancer cells migrate through

Correspondence to: Dr Guo-Qing Chen, Department of Surgery, Shanghai Tongren Hospital of Changning, 768 Yuyuan Road, Shanghai 200050, P.R. China

E-mail: cguoqing@yeah.net

${ }^{*}$ Contributed equally

Key words: TET1, HDAC inhibitor, TSA, breast cancer the extracellular matrix from the primary tumor, which is associated with the upregulated expression of matrix metalloproteinases (MMPs) (2-4). Therefore, it is important to elucidate the mechanisms underlying cancer invasion.

Histone acetylation status regulated by histone acetyltransferases (HATs) and histone deacetylases (HDACs) is important in the regulation of gene expression by affecting chromatin structure and accessibility $(5,6)$. HDACs are recruited to DNA-bound transcription factors resulting in the removal of acetyl groups from nucleosomal histones or directly interact with transcription factors to modulate gene expression (7-10). HDAC inhibition leads to the accumulation of acetylation in histones and transcription factors, and specifically programmed gene expression patterns $(11,12)$.

In humans, the reduction of histone acetylation is significantly associated with tumor progression and invasion (13). Emerging evidence indicates that histone deacetylase inhibitors (HDACi) induce growth inhibition, cell cycle arrest and programmed cell death in diverse cancer cells $(14,15)$. HDACi treatment upregulates the expression of suppressors of metastasis and downregulates invasion-promoting genes, resulting in the repression of cancer cell invasion and metastasis (16). HDAC inhibition is emerging as a potential strategy for cancer therapy and several HDACi have been developed for clinical trials in patients with solid malignancies (17). Trichostatin A (TSA), a non-competitive reversible inhibitor of HDAC activity, has been reported to inhibit cancer invasion and metastasis in vivo and in vitro (18-20). HDACi modulate cancer progression through affecting the acetylation of histone and non-histone proteins to reactivate the transcription of differential target genes. It has been proposed that TSA upregulates RECK to suppress MMP2 activation and cancer cell invasion (21). Therefore, it is important to investigate the targets of HDACi in order to elucidate the molecular mechanisms underlying HDACi elicited phenotypes.

In the present study, TSA was observed to inhibit cell invasion in MCF-7 breast cancer cells. Furthermore, the expression of tissue inhibitors of metalloproteinase 2/3 (TIMP2/3) was 
upregulated and the expression of MMP2/9 was decreased by TSA treatment. Notably, TIMP2/3 and MMP2/9 have been revealed as targets of TET1 in prostate and breast cancer invasion (22). Additionally, tumor development is associated with a decrease in TET expression and 5-methylcytosine hydroxylation (23). TIMP2 and TET1 consistently demonstrated downregulation during breast cancer progression in vivo. Our hypothesis was that TET1 may be one of the targets of HDACi in breast cancer invasion. As expected, TET1 was upregulated by TSA stimulation and TET1 knockdown facilitated breast cancer cell invasion. Importantly, TET1 depletion impaired TSA induced suppression of cell invasion, suggesting that TET1 may act as one of the HDACi targets partially mediating TSA elicited anti-cancer activity.

\section{Materials and methods}

Patient samples and cell culture. The present study was approved by the ethics committee of Shanghai Tongren Hospital (Shanghai, China), and written informed consent was obtained from all participants prior to the study. A total of 61 cancer specimens from breast cancer patients from stages I to IV were collected and the conditions of these patients are summarized in Table I. Samples were individually fresh-frozen in TRIzol reagent (Invitrogen Life Technologies, Carlsbad, CA, USA). All patients were histologically examined at Shanghai Tongren Hospital (Shanghai, China) and written informed consent was obtained from all study participants. Breast cancer MCF-7 cells were cultured in RPMI-1640 medium (Invitrogen Life Technologies) supplemented with $10 \%$ fetal calf serum at $37^{\circ} \mathrm{C}$ and $5 \% \mathrm{CO}_{2}$. TSA dissolved in DMSO was applied in the present study with the indicated concentrations.

Wound-healing assay. Wound healing was performed as previously described in 12-well plates (24). Briefly, after $\mathrm{MCF}-7$ cells grew to $>90 \%$ confluence, a wound was introduced using a $200 \mu \mathrm{l}$ pipette tip. Subsequently, the cells were washed once with PBS and then incubated at $37^{\circ} \mathrm{C}$ and $5 \%$ $\mathrm{CO}_{2}$. Wound closure was monitored over an indicated time period and images were captured at the four intersecting edges of the cross. Wound width at 0 and 24 or $36 \mathrm{~h}$ was measured and the difference plotted as the percentage of wound closure.

Quantitative real-time PCR ( $q R T-P C R)$. Total RNA was extracted from MCF-7 cells or cancer tissues using TRIzol reagent (Invitrogen Life Technologies). For each sample, 2.5 or $5 \mu \mathrm{g}$ of total RNA was reverse transcribed using the QuantiTect ReverseTranscriptionkit(Qiagen,Hilden,Germany).ThecDNA product was then quantified by SYBR-Green real-time PCR master mix (Toyobo, Osaka, Japan). The primers used were as follows: TET1, forward 5'-GAGCCTGTTCCTCGATGTGG-3' and reverse 5'-CAAACCCACCTGAGGCTGTT-3'; GAPDH, forward 5'-GTGTTCCTACCCCCAATGTGT-3' and reverse 5'-ATTGTCATACCAGGAAATGAGCTT-3'; TIMP2, forward 5'-GGGTCTCGCTGGACATTG-3' and reverse 5'-TTGATGTTCTTCTCCGTGACC-3'; TIMP3, forward 5'-CATGTGCAGTACATCCATACGG-3' and reverse 5'-CATCATAGACGCGACCTGTCA-3'; MMP2, forward 5'-AAGGCCAAGTGGTCCGTGTGAA-3' and reverse 5'-AACAGTGGACATGGCGGTCTCAG-3'; MMP9,
Table I. Characteristics of breast cancer patients.

\begin{tabular}{lcr}
\hline & \multicolumn{2}{c}{ Age (years) } \\
\cline { 2 - 3 } Phase & $40-49$ & $50+$ \\
\hline I & 5 & 7 \\
II & 12 & 14 \\
III & 7 & 9 \\
IV & 3 & 4
\end{tabular}

forward 5'-CACGTCCACCCCTCAGAGC-3' and reverse 5'-GCCACTTGTCGGCGATAAGC-3'.

Western blot analysis. MCF-7 cells were lysed with two-fold loading buffer [20 mM of Tris- $\mathrm{HCl}$ (pH 7.4), $2 \mathrm{mM}$ of EDTA and $1 \%$ Triton $\mathrm{X}-100$ ] and the supernatant was subjected to western blot analysis, which was performed as previously described (25). GAPDH was used as the loading control. The following primary antibodies were used: anti-TET1 (Abcam, Cambridge, UK; 1:500), anti-MMP2 (Abcam; 1:400), anti-TIMP2 (Abcam; 1:500) and anti-GAPDH (Millipore, Billerica, MA, USA; 1:10,000).

shRNA and transfection. Chemically synthesized TET1 shRNA and scramble shRNA (control) were annealed and cloned into a short interfering RNA expressing vector named pSUPER (Oligoengine, Seattle, WA, USA). Control or TET1 shRNA targeted sequences were: control shRNA 5'-GCTACGAAGCACCTCTCTTAG-3' and TET1 shRNA 5'-CGATGCAAGCCATCCTTTCGA-3'. Plasmids purified by the Qiagen purification kit were transfected into MCF-7 cells at 40-60\% confluency with Lipofectamine 2000 (Invitrogen Life Technologies) according to the manufacturer's instructions.

Statistic analysis. SPSS 11.0 software was used for statistical analysis in the present study. The values are presented as the mean \pm SD and one-way ANOVA was applied for group differences. All tests were two-sided and $\mathrm{P}<0.05$ was considered to indicate a statistically significant difference.

\section{Results}

HDACi suppresses breast cancer cell invasion and regulates TIMP2/3 and MMP2/9 expression. Previously, HDACi were reported to inhibit breast cancer cell invasion $(18,26)$. Prior to examining the mechanisms of HDAC in cancer invasion, the effects of TSA on cell invasion were examined by a wound-healing assay in MCF-7 breast cancer cells. Consistent with previous findings, TSA significantly suppressed cell invasion (Fig. 1A and B). TIMP2 and 3, the important suppressors of cancer cell invasion $(27,28)$, were upregulated under the stimulation of TSA. By contrast, the expression of MMP2/9, which was correlated with clinicopathological disease variables in breast cancer $(29,30)$, was increased by HDAC inhibition (Fig. 1C). Furthermore, TSA affected MMP2 and TIMP2 expression and this was verified at the protein level 
A

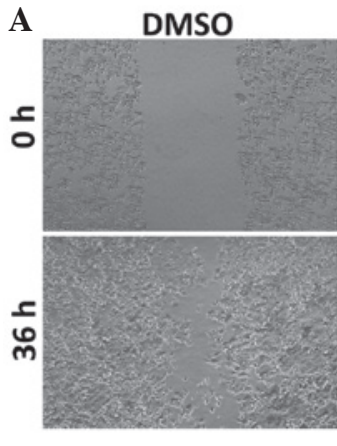

C

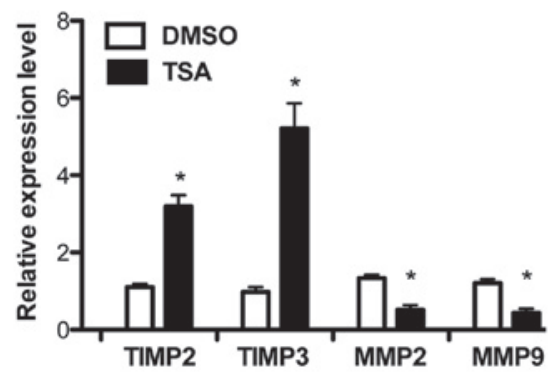

B

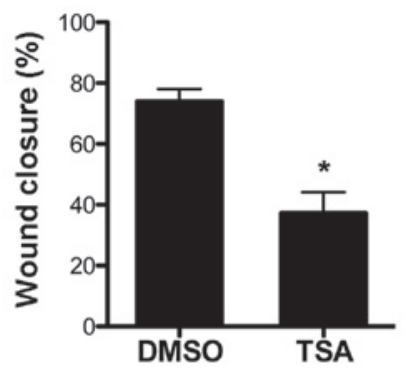

D

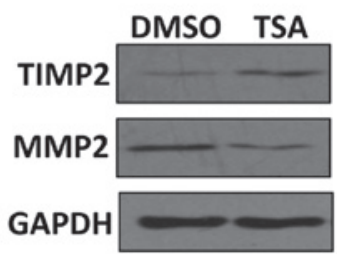

Figure 1. TSA suppresses breast cancer cell invasion and regulates TIMP2/3 and MMP2/9 expression. (A) Wound-healing assay was performed in MCF-7 cells when confluent monolayers of MCF-7 cells were wounded using a pipette tip. DMSO or $10 \mathrm{ng} / \mathrm{ml}$ of TSA were supplemented into wounded cells for $36 \mathrm{~h}$. Wound closure was monitored by microscopy and representative photomicrographs are shown. (B) Wound width was calculated at 0 and $36 \mathrm{~h}$, and the difference was plotted as the percentage of wound closure. The values shown are the mean \pm SEM of three independent experiments. (C and D) Expression of TIMP2, TIMP3, MMP2 and MMP9 was examined by qRT-PCR in DMSO or TSA treated MCF-7 cells at $36 \mathrm{~h}$. (D) The protein level of TIMP2 and MMP2 was also determined by western blot analysis. " $\mathrm{P}<0.05$ vs. DMSO. TSA, trichostatin A; TIMP2/3, tissue inhibitors of metalloproteinase 2/3; MMP2/9, matrix metalloproteinases 2/9; qRT-PCR, quantitative real-time PCR.
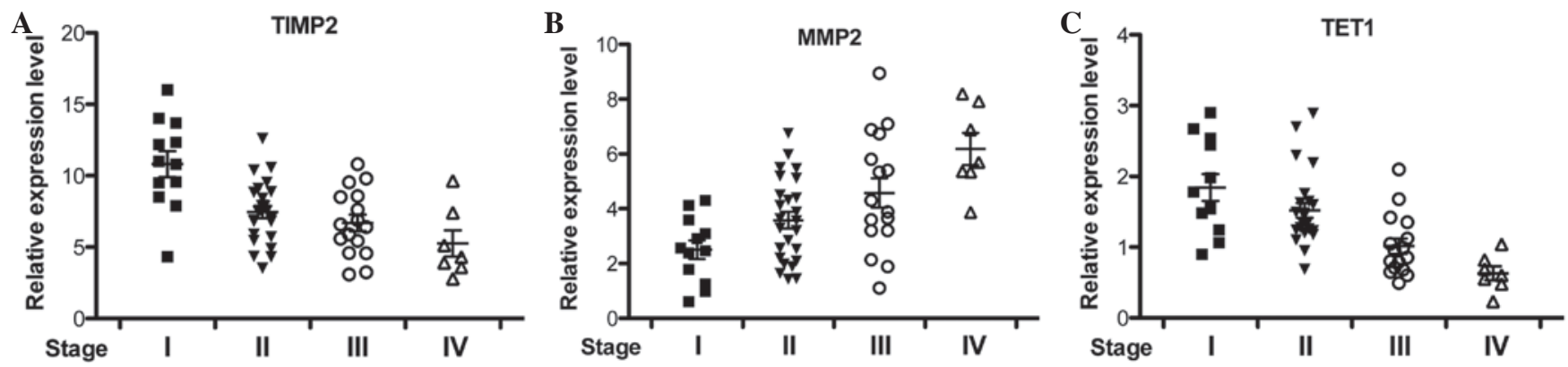

Figure 2. TIMP2, MMP2 and TET1 expression during breast cancer development. qRT-PCR was performed to detect the expression levels of (A) TIMP2, (B) MMP2 and (C) TET1 in multiple breast cancer patients with different stages of tumors (stage I, n=12; stage II, n=26; stage III, n=16; stage IV, n=7). TIMP2, tissue inhibitors of metalloproteinase 2; MMP2, matrix metalloproteinase 2; qRT-PCR, quantitative real-time PCR.

(Fig. 1D). In addition, MMP2/9 and TIMP2/3 mRNA levels were not affected when TSA was administered to cells within $2 \mathrm{~h}$ (data not shown). This demonstrates that TIMP $2 / 3$ and MMP2/9 expression may be indirectly modulated by HDAC in breast cancer.

TIMP2, MMP2 and TET1 expression during breast cancer development. Coincidently, TIMPs and MMPs were revealed as downstream targets of TET1 and TET1 suppresses invasion partly through TIMP activation and MMP inhibition in prostate and breast cancer (22). In order to investigate the correlation between TIMP2, MMP2 and TET1 in vivo, the expression of these genes in multiple breast cancer patients at different stages was examined by qRT-PCR. TIMP2 and MMP2 expression was progressively downregulated and upregulated with breast cancer development, respectively (Fig. 2A and B).
Consistently, TET1 mRNA level was reduced in breast cancer tissues (Fig. 2C), which corrrelated with TIMP2 expression changes (23). Collectively, TIMP2 and TET1 downregulation and MMP2 upregulation were correlated with breast cancer progression.

TET1 knockdown facilitates breast cancer cell invasion. Subsequently, the present study examined whether the reduction of TET1 is functionally involved in breast cancer cell invasion. TET1 shRNA was delivered into MCF-7 cells to efficiently knockdown TET1 expression (Fig. 3A). Then the effects of TET1 knockdown on cell invasion were analyzed and the results demonstrated that TET1 depletion by TET1 shRNA increased the cell invasion capacity (Fig. 3B and C). Notably, TET1 knockdown resulted in a decrease in TIMP2/3 and the upregulation of MMP2/9 expression (Fig. 3D), which 
A
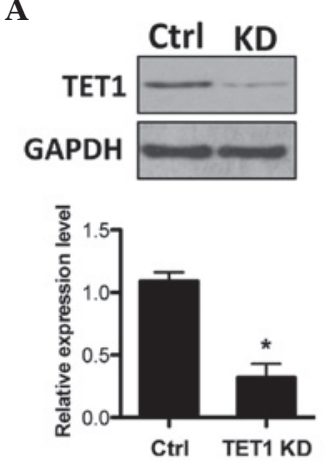

C

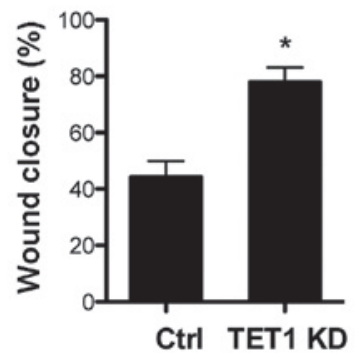

B
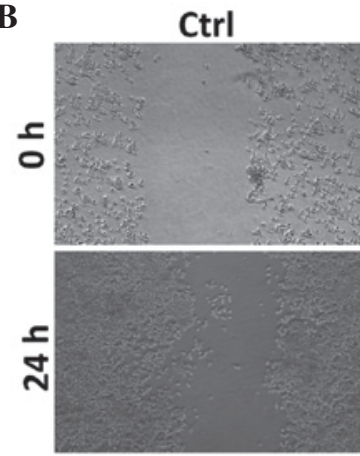

D

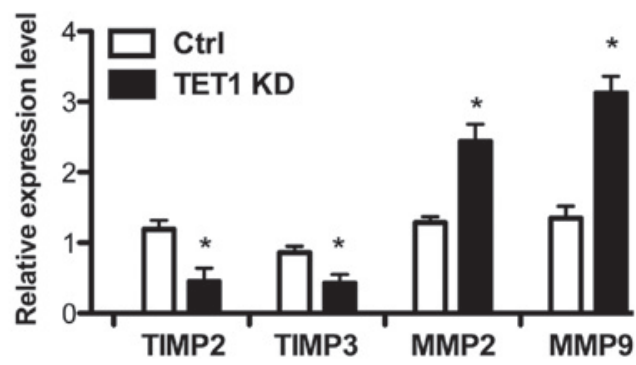

Figure 3. TET1 knockdown facilitates breast cancer cell invasion. (A) TET1 protein and mRNA levels were determined in Ctrl or TET1 KD transfected MCF-7 cells by western blot analysis and qRT-PCR, respectively. GAPDH served as the loading control. (B and C) Wound-healing assay was performed in the Ctrl or TET1 KD expressing MCF-7 cells. (B) Wound closure was captured by microscopy following wounding for $24 \mathrm{~h}$ and (C) quantified. (D) Relative expression levels of TIMP2, TIMP3, MMP2 and MMP9 were checked by qRT-PCR in the Ctrl or TET1 KD expressing MCF-7 cells. "P<0.05 vs. CTRL. qRT-PCR, quantitative real-time PCR; TIMP, tissue inhibitors of metalloproteinase; MMP, matrix metalloproteinase; Ctrl, control shRNA; KD, knockdown shRNA.

A

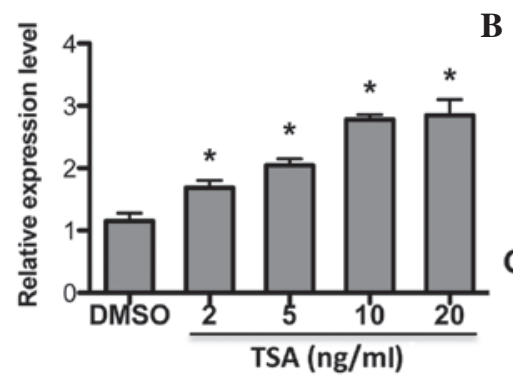

B

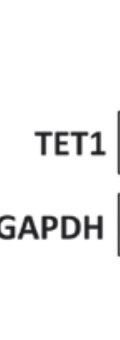

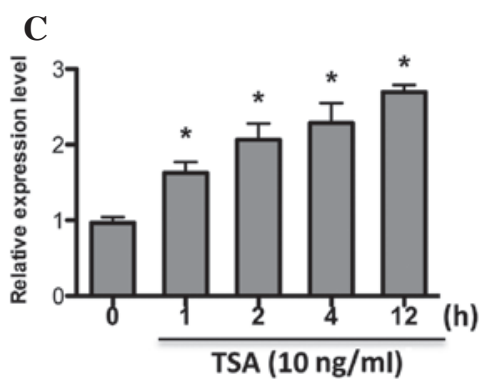

Figure 4. TSA upregulates TET1 expression in MCF-7 cells. (A) TET1 mRNA level was examined by qRT-PCR in MCF-7 cells in the presence of 2, 5, 10 and $20 \mathrm{ng} / \mathrm{ml}$ of TSA for $24 \mathrm{~h}$. DMSO was added into the medium as the control. (B) Western blot analysis was performed to determine the protein level of TET1 and GAPDH in MCF-7 cells under the condition in (A). (C) MCF-7 cells were treated with $10 \mathrm{ng} / \mathrm{ml}$ of TSA for 0, 1, 2, 4 and $12 \mathrm{~h}$ and the TET1 mRNA level was determined. "P<0.05 vs. DMSO. TSA, trichostatin A; qRT-PCR, quantitative real-time PCR.

is opposite to the effects of TSA in MCF-7 cells (Fig. 1A). This indicates that TET1 suppresses breast cancer cell invasion.

TSA upregulates TET1 expression in MCF-7 cells. Given that the expression of TIMPs and MMPs was affected by HDAC inhibition and TET1, our hypothesis was that TET1 may be regulated by HDACi. As expected, TET1 expression was upregulated by TSA in a dose-dependent manner (Fig. 4A), which was confirmed by western blot analysis (Fig. 4B). Additionally, TET1 was responsive to short-term TSA treatment (Fig. 4C). This suggests that TET1 may be a target of TSA in breast cancer.

TET1 knockdown impairs TSA-induced suppression of breast cancer cell invasion. Since TET1 expression was found to be regulated by HDAC inhibition, the functional association between TSA and TET1 needed to be elucidated. In order to examine whether TET1 is able to mediate TSA induced suppression of breast cancer cells, TSA was subjected to TET1-knockdown and cell invasion was examined. It was revealed that the inhibitory effect of TSA on breast cancer cell invasion was impaired in TET1-knockdown cells when compared with control shRNA expressing cells (Fig. 5A and B). Correspondingly, TSA upregulated TIMP2/3 and downregulated MMP2/9 expression, which were also impaired in TET1-knockdown cells. This indicates that TET1 partially mediates TSA induced repression of breast cancer cell invasion.

\section{Discussion}

Breast cancer is one of the most common types of cancer and has a high risk of mortality among females all over the world (31). Therefore there is a great need for understanding 

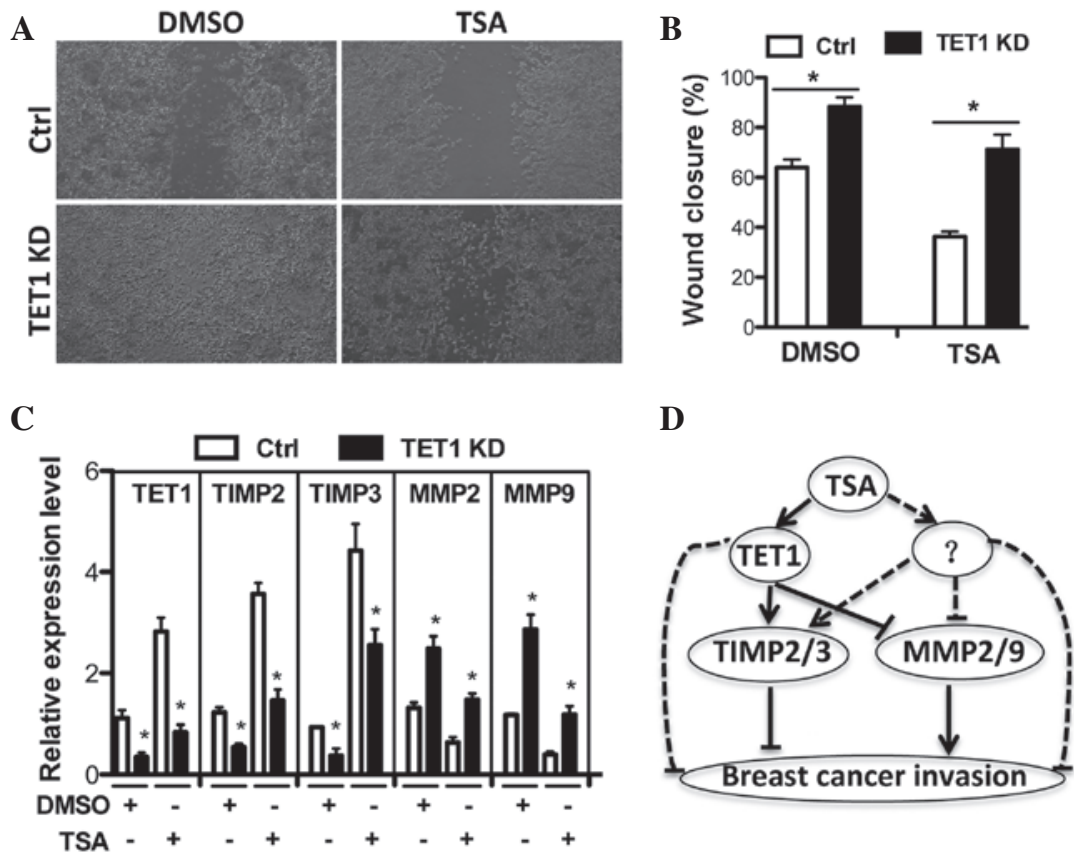

Figure 5. TET1 partially mediates TSA-induced suppression of breast cancer cell invasion. (A-B) Ctrl or TET1 KD expressing MCF-7 cells were treated with DMSO or $10 \mathrm{ng} / \mathrm{ml}$ of TSA for $36 \mathrm{~h}$ after wounding. (A) Wound closure was monitored and (B) quantified. (C) Expression levels of TET1, TIMP2, TIMP3, MMP2 and MMP9 were measured by qRT-PCR in Ctrl or TET1 KD MCF-7 cells under the condition in (A). (D) Hypothetical model for the action mechanism of TSA in the suppression of breast cancer cell invasion. Briefly, TSA regulates TET1 expression or other unknown factors to promote TIMP2/3 expression and inhibit MMP2/9 transcription leading to the suppression of breast cancer cell invasion. "P<0.05 vs. CTRL. TSA, trichostatin A; Ctrl, control shRNA; KD, knockdown shRNA; qRT-PCR, quantitative real-time PCR; TIMP, tissue inhibitors of metalloproteinase; MMP, matrix metalloproteinase.

the molecular mechanisms underlying cancer progression and for the development of more effective therapeutic strategies for breast cancer. Carcinogenesis is able to be regulated by genetic and epigenetic alterations and epigenetic alterations are a reversible process, which makes epigenetic modifications preferable for clinical therapy $(32,33)$.

DNA methylation and histone modifications are the most important epigenetic mediators of transcriptional regulation and multiple HDACi are in clinical development for hematological and solid tumor treatment $(34,35)$. HDACi result in the accumulation of acetylation in histones and non-histone proteins to modulate target gene expression in cancerous cells, which reactivates the expression of tumor suppressors (35). In the present study (Fig. 1) and previous studies $(18,36)$, the non-competitive reversible HDACi TSA has been demonstrated to suppress breast cancer cell invasion. The limitations of HDAC inhibition in cancer therapy is the non-specificity and toxicity of the chemical inhibitors. It is important to understand the molecular mechanisms in HDACi treatment. The HDACi valproic acid (VPA) induces ER $\alpha$ expression in its anti-tumor effects (37) and TSA enhances the acetylation and stability of the ER $\alpha$ and $\mathrm{p} 300$ proteins that may contribute to breast cancer treatment (38). TSA also directly upregulates p53 and RECK, which are important in tumor suppression $(21,39)$.

The present study found that TIMP2/3 were increased and that MMP2/9 were decreased when treated with TSA (Fig. 1). It has been proposed that TET1 suppresses breast cancer invasion through the activation of TIMPs and the inhibition of MMPs (22). Additionally, TET1 and TIMP2/3 reduction was inversely correlated with MMP2/9 upregulation during breast cancer progression (Fig. 2) (23). Notably, TET1 demonstrated similar functions in MCF-7 breast cancer cell invasion (Fig. 3) (22). It is reasonable to postulate that TSA may inhibit breast cancer invasion through the regulation of TET1 expression. Notably, TSA promoted TET1 expression in a dose-dependent manner (Fig. 4) and TET1 partially mediated TSA elicited suppression of cell invasion (Fig. 5), which was in accordance with our hypothesis. TET1 expression may be negatively controlled by a specific HDAC and TSA treatment releases the HDAC inhibition on TET1 expression. TET1 was found to be associated with TIMPs genes and promoter regions to regulate their DNA methylation status and transcription levels $(22,40)$. Our hypothesis is that TSA may indirectly affect 5-methylcytosine hydroxylation of TIMP gene promoters through the regulation of TET1 expression, which will be investigated in our future study. There is a possibility that VPA enhances the global 5-methylcytosine level in nuclear DNA (41).

Furthermore, the alteration in DNA methylation status is another important epigenetic modification and DNA methyltransferases (DNMTs) have become an epigenetic therapy target in various types of cancer (42). 5-azacytidine, a global DNMT inhibitor, has been approved for clinical trials against solid tumors (43). Currently, synergistic treatment with DNMT and HDACi has been applied for producing optimal effects (41).

As summarized in Fig. 5D, the HDACi TSA may upregulate the expression of TET1, which results in the activation of TIMPs and the inhibition of MMPs, thus, leading to the suppression of breast cancer cell invasion. The present study provides a novel molecular mechanism for HDAC inhibition in tumor suppression. This may provide insights into the role of HDACs in cancer development and epigenetic therapy. 


\section{Acknowledgements}

We would like to thank Shanghai Xuhai Biological Technology Co., Ltd for the experimental support.

\section{References}

1. Steeg PS: Metastasis suppressors alter the signal transduction of cancer cells. Nat Rev Cancer 3: 55-63, 2003.

2. Mohanam S, Sawaya R, McCutcheon I, Ali-Osman F, Boyd D and Rao JS: Modulation of in vitro invasion of human glioblastoma cells by urokinase-type plasminogen activator receptor antibody. Cancer Res 53: 4143-4147, 1993.

3. Rao JS: Molecular mechanisms of glioma invasiveness: the role of proteases. Nat Rev Cancer 3: 489-501, 2003.

4. Davies B, Waxman J, Wasan H, Abel P, Williams G, Krausz T, Neal D, Thomas D, Hanby A and Balkwill F: Levels of matrix metalloproteases in bladder cancer correlate with tumor grade and invasion. Cancer Res 53: 5365-5369, 1993.

5. Horikoshi M: Histone acetylation: from code to web and router via intrinsically disordered regions. Curr Pharm Des 19: 5019-5042, 2013.

6. Gräff J and Tsai LH: Histone acetylation: molecular mnemonics on the chromatin. Nat Rev Neurosci 14: 97-111, 2013.

7. Mayo MW, Denlinger CE, Broad RM, Yeung F, Reilly ET, Shi Y and Jones DR: Ineffectiveness of histone deacetylase inhibitors to induce apoptosis involves the transcriptional activation of NF-kappa B through the Akt pathway. J Biol Chem 278: 18980-18989, 2003.

8. Brochier C, Dennis G, Rivieccio MA, McLaughlin K, Coppola G Ratan RR and Langley B: Specific acetylation of p53 by HDAC inhibition prevents DNA damage-induced apoptosis in neurons. J Neurosci 33: 8621-8632, 2013.

9. Sachweh MC, Drummond CJ, Higgins M, Campbell J and Laín S: Incompatible effects of p53 and HDAC inhibition on p21 expression and cell cycle progression. Cell Death Dis 4: e533, 2013.

10. Cheung WL, Briggs SD and Allis CD: Acetylation and chromosomal functions. Curr Opin Cell Biol 12: 326-333, 2000.

11. Van Lint C, Emiliani S and Verdin E: The expression of a small fraction of cellular genes is changed in response to histone hyperacetylation. Gene Expr 5: 245-253, 1996.

12. Mitsiades CS, Mitsiades NS, McMullan CJ, et al: Transcriptional signature of histone deacetylase inhibition in multiple myeloma: biological and clinical implications. Proc Natl Acad Sci USA 101: 540-545, 2004

13. Song J, Noh JH, Lee JH, et al: Increased expression of histone deacetylase 2 is found in human gastric cancer. APMIS 113 264-268, 2005

14. Shao Y, Gao Z, Marks PA and Jiang X: Apoptotic and autophagic cell death induced by histone deacetylase inhibitors. Proc Nat Acad Sci USA 101: 18030-18035, 2004.

15. Marks PA, Richon VM, Miller T and Kelly WK: Histone deacetylase inhibitors. Adv Cancer Res 91: 137-168, 2004.

16. McGarry LC, Winnie JN and Ozanne BW: Invasion of $\mathrm{v}$-Fos(FBR)-transformed cells is dependent upon histone deacetylase activity and suppression of histone deacetylase regulated genes. Oncogene 23: 5284-5292, 2004.

17. Marks PA, Richon VM, Kelly WK, Chiao JH and Miller T: Histone deacetylase inhibitors: development as cancer therapy. Novartis Found Symp 259: 269-281, 2004.

18. Vigushin DM, Ali S, Pace PE, et al: Trichostatin A is a histone deacetylase inhibitor with potent antitumor activity against breast cancer in vivo. Clin Cancer Res 7: 971-976, 2001

19. Tarasenko N, Nudelman A, Tarasenko I, et al: Histone deacetylase inhibitors: the anticancer, antimetastatic and antiangiogenic activities of AN-7 are superior to those of the clinically tested AN-9 (Pivanex). Clin Exp Metastasis 25: 703-716, 2008.

20. Bolden JE, Peart MJ and Johnstone RW: Anticancer activities of histone deacetylase inhibitors. Nat Rev Drug Discov 5: 769-784 2006.

21. Liu LT, Chang HC, Chiang LC and Hung WC: Histone deacetylase inhibitor up-regulates RECK to inhibit MMP-2 activation and cancer cell invasion. Cancer Res 63: 3069-3072, 2003.
22. Hsu CH, Peng KL, Kang ML, et al: TET1 suppresses cancer invasion by activating the tissue inhibitors of metalloproteinases. Cell Rep 2: 568-579, 2012.

23. Yang H, Liu Y, Bai F, et al: Tumor development is associated with decrease of TET gene expression and 5-methylcytosine hydroxylation. Oncogene 32: 663-669, 2013.

24. Asrani K, Keri RA, Galisteo R, et al: The HER2- and heregulin beta1 (HRG)-inducible TNFR superfamily member Fn14 promotes HRG-driven breast cancer cell migration, invasion, and MMP9 expression. Mol Cancer Res 11: 393-404, 2013.

25. Brown SA, Ghosh A and Winkles JA: Full-length, membrane-anchored TWEAK can function as a juxtacrine signaling molecule and activate the NF-kappaB pathway. J Biol Chem 285: 17432-17441, 2010.

26. Grimaldi C, Pisanti S, Laezza C, et al: Anandamide inhibits adhesion and migration of breast cancer cells. Exp Cell Res 312: 363-373, 2006.

27. Albini A, Melchiori A, Santi L, Liotta LA, Brown PD and Stetler-Stevenson WG: Tumor cell invasion inhibited by TIMP-2. J Natl Cancer Inst 83: 775-779, 1991.

28. Baker AH, George SJ, Zaltsman AB, Murphy G and Newby AC: Inhibition of invasion and induction of apoptotic cell death of cancer cell lines by overexpression of TIMP-3. Br J Cancer 79: 1347-1355, 1999.

29. Jinga D, Stefanescu M, Blidaru A, Condrea I, Pistol G and Matache C: Serum levels of matrix metalloproteinases MMP-2 and MMP-9 and their tissue natural inhibitors in breast tumors. Roum Arch Microbiol Immunol 63: 141-158, 2004.

30. Sier CF, Kubben FJ, Ganesh S, et al: Tissue levels of matrix metalloproteinases MMP-2 and MMP-9 are related to the overall survival of patients with gastric carcinoma. Br J Cancer 74: 413-417, 1996.

31. Jemal A, Siegel R, Ward E, et al: Cancer statistics, 2008. CA Cancer J Clin 58: 71-96, 2008.

32. Taby R and Issa JP: Cancer epigenetics. CA Cancer J Clin 60: 376-392, 2010.

33. Cai FF, Kohler C, Zhang B, Wang MH, Chen WJ and Zhong XY: Epigenetic therapy for breast cancer. Int J Mol Sci 12: 4465-4487, 2011.

34. Namdar M, Perez G, Ngo L and Marks PA: Selective inhibition of histone deacetylase 6 (HDAC6) induces DNA damage and sensitizes transformed cells to anticancer agents. Proc Natl Acad Sci USA 107: 20003-20008, 2010.

35. Liu T, Kuljaca S, Tee A and Marshall GM: Histone deacetylase inhibitors: multifunctional anticancer agents. Cancer Treat Rev 32: 157-165, 2006

36. Yang X, Phillips DL, Ferguson AT, Nelson WG, Herman JG and Davidson NE: Synergistic activation of functional estrogen receptor (ER)-alpha by DNA methyltransferase and histone deacetylase inhibition in human ER-alpha-negative breast cancer cells. Cancer Res 61: 7025-7029, 2001.

37. Travaglini L, Vian L, Billi M, Grignani F and Nervi C: Epigenetic reprogramming of breast cancer cells by valproic acid occurs regardless of estrogen receptor status. Int J Biochem Cell Biol 41: 225-234, 2009

38. Kim SH, Kang HJ, Na H and Lee MO: Trichostatin A enhances acetylation as well as protein stability of ERalpha through induction of p300 protein. Breast Cancer Res 12: R22, 2010.

39. Henderson C, Mizzau M, Paroni G, Maestro R, Schneider C and Brancolini C: Role of caspases, Bid, and p53 in the apoptotic response triggered by histone deacetylase inhibitors trichostatin-A (TSA) and suberoylanilide hydroxamic acid (SAHA). J Biol Chem 278: 12579-12589, 2003.

40. Williams K, Christensen J, Pedersen MT, et al: TET1 and hydroxymethylcytosine in transcription and DNA methylation fidelity. Nature 473: 343-348, 2011

41. Kristensen LS, Nielsen HM and Hansen LL: Epigenetics and cancer treatment. Eur J Pharmacol 625: 131-142, 2009.

42. Hatziapostolou M and Iliopoulos D: Epigenetic aberrations during oncogenesis. Cell Mol Life Sci 68: 1681-1702, 2011.

43. Chik F and Szyf M: Effects of specific DNMT gene depletion on cancer cell transformation and breast cancer cell invasion; toward selective DNMT inhibitors. Carcinogenesis 32: 224-232, 2011. 